\title{
Contingency Trajectory Design for a Lunar Orbit Insertion Maneuver Failure by the LADEE Spacecraft
}

\author{
A. L. Genova* \\ NASA Ames Research Center, Moffett Field, CA, 94035, USA
}

\begin{abstract}
A contingency trajectory analysis was performed for NASA Ames Research Center's (ARC's) Lunar Atmosphere and Dust Environment Explorer (LADEE) spacecraft in case of a missed lunar orbit insertion (LOI) maneuver. Recovery trajectory options are shown to exist for all LADEE launch opportunities throughout a one year period. Recovery $\Delta V$ costs primarily depended on the spacecraft's apogee location on or near the Sun-Earth weak stability boundary (WSB) and the time needed by the spacecraft to recover (e.g. to "wake up" from "safe" mode) to perform an escape prevention maneuver after the missed LOI.
\end{abstract}

\begin{tabular}{|c|c|}
\hline & Nomenclature \\
\hline$\Delta V$ & $=$ delta- $\mathrm{V}$, change in velocity $(\mathrm{m} / \mathrm{s})$ \\
\hline Apogee & $=$ spacecraft's farthest point from Earth while in orbit \\
\hline C3 & $=($ Earth $)$ orbit Keplerian energy or the square of relative asymptotic (Earth) velocity $\left(\mathrm{km}^{2} / \mathrm{s}^{2}\right)$ \\
\hline$I P$ & $=$ in-plane, with regard to a nominal solution's inclination in Earth's equatorial plane \\
\hline$L O I$ & $=$ lunar orbit insertion $(\mathrm{m} / \mathrm{s})$ \\
\hline$O P$ & $=$ out-of-plane, with regard to a nominal solution's inclination in Earth's equatorial plane \\
\hline Perigee & $=$ spacecraft's point of closest Earth approach while in orbit \\
\hline$W S B$ & $\begin{array}{l}=\text { weak stability boundary: First mentioned as "stability boundary" by Belbruno }{ }^{1} \text { and changed to } \\
\text { "weak stability boundary"2,3 (alternately known as "fuzzy boundary"4), the WSB is a complex } \\
\text { region (and fractal set) in six dimensional space and is the approximate transition region } \\
\text { between negative (temporary capture; stable set) and positive (escape; unstable set) Keplerian } \\
\text { orbit energy (i.e., C3) with respect to the primary body. The WSB can be represented by } \\
\text { invariant manifolds and approximated by zero-velocity curves }{ }^{5-11} \text {. }\end{array}$ \\
\hline
\end{tabular}

\section{Introduction}

TN the event of a missed lunar orbit insertion (LOI) maneuver by the Lunar Atmosphere and Dust Environment 1 Explorer (LADEE) spacecraft, the author was responsible for designing a trajectory that would recover the spacecraft into its intended near-equatorial, circular retrograde lunar science orbit. Universe Today reported that LADEE's LOI consisted of "... absolutely critical do or die orbital insertion engine firings" stated that if LOI was missed, LADEE would have “...passed the Moon with no hope of returning." receiving LADEE's planned LOI state vector two weeks before the actual LOI, the author designed a viable rescue trajectory that was verified by the LADEE flight dynamics team and flight-ready more than ten days before LOI. Fortunately the LADEE spacecraft, built and operated by NASA Ames Research Center (ARC), did not fly this recovery trajectory, since the actual LOI was successfully performed on Oct. 6, 2013, notably via a skeleton crew during the U.S. Government Shutdown of 2013 (Oct. 1 to 16). Details of the selected contingency trajectory design and other considered designs are presented herein.

\footnotetext{
* Trajectory Designer, Mission Design Division, NASA Ames Research Center, MS202-1, AIAA member

${ }^{\dagger}$ Universe Today, "Skeleton Crew gets LADEE in Orbit, Checked out and Fires Revolutionary Laser During Gov't Shutdown", Oct. 20, 2014, URL: http://www.universetoday.com/105630/skeleton-crew-gets-ladee-in-orbit-checkedout-and-fires-revolutionary-laser-during-govt-shutdown [cited June 22, 2014]

* Spaceflight101, "LADEE in Lunar Orbit after Successful LOI Maneuver", Oct. 6, 2013, URL: http://www.spaceflight101.com/ladee-mission-updates.html [cited June 22, 2014]
} 


\section{Assumptions and Constraints}

The presented trajectory design was performed primarily using the Systems Tool Kit (STK) Astrogator module. A seventh order Runge-Kutta-Fehlberg numerical integrator with eighth order error control was used for orbit propagation. The force model included solar radiation pressure (SRP), a Jacchia-Roberts Earth atmosphere model, and gravity field models of the Earth (30 by 30), Moon (30 by 30), and Sun (four by zero). Maneuvers were assumed to be impulsive. The total available recovery $\Delta \mathrm{V}$ was constrained to less than $860 \mathrm{~m} / \mathrm{s}$ for three months of nominal science operations. LADEE's nominal science orbit was a $250 \mathrm{~km}$ circular (with an initial perilune altitude of $587 \mathrm{~km}$ ), retrograde lunar orbit with inclination of 157 degrees to obtain required dust measurements at low lunar altitudes, passing from darkness into daylight over the lunar terminator. DE421 was the ephemeris source used for both the Earth and Moon. LADEE's state vector at the time of planned LOI (Julian Date 2456571.9531057) using the Earth J2000 Cartesian coordinate system: $\left[\mathrm{x}, \mathrm{y}, \mathrm{z}, \mathrm{V}_{\mathrm{x}}, \mathrm{V}_{\mathrm{y}}, \mathrm{V}_{\mathrm{z}}\right]=[-324311 \mathrm{~km},-176241 \mathrm{~km},-81134.7 \mathrm{~km}$, $1.02087 \mathrm{~km} / \mathrm{s}, 0.78829 \mathrm{~km} / \mathrm{s}, 1.22223 \mathrm{~km} / \mathrm{s}]$.

\section{Contingency Trajectory Design and Analysis}

Presented herein are several LOI recovery trajectory design types considered for use by the LADEE spacecraft.

\section{LADEE Mission Nominal Trajectory}

The LADEE spacecraft's nominal trajectory is seen in Fig. 1a (from Ref. 12), from launch on Sep. 5, 2013 to LOI on Oct. 6, 2013. This atypical monthlong lunar transfer trajectory implemented eccentric Earth phasing orbits with apogee altitudes ranging from about 275,000 km (seven day period) to lunar distance (ten day period) and was flown by LADEE for multiple reasons including: 1) The launch vehicle (LV), a Minotaur-V launched from Wallops, VA could not send the LADEE spacecraft's $383 \mathrm{~kg}$ initial mass all the way to the Moon; 2) The LV's fifth stage injection accuracy was not expected to be as high as that of other larger LVs, thus the longer lunar transfer allowed ample time for the spacecraft to perform trajectory correction maneuvers to correct LV injection errors; 3) The launch window could be lengthened by varying the phasing orbits' periods ${ }^{12}$.

LADEE considered two types of nominal lunar transfers for a given launch month, termed in-plane (IP) and out-of-plane (OP). IP solutions were generally less inclined to the Earth equatorial and lunar equatorial planes (vs. OP solutions), (Fig. 1d); however OP solutions provided better solar lighting conditions for the spacecraft throughout the sub-lunar Earth phasing orbits and were thus preferred over IP solutions by the LADEE team.

Both solution types were propagated to the Earth-Moon WSB after the LOI miss throughout a July 2013 to June 2014 launch period. It was seen that OP solutions were in lower energy (C3) Earth orbits than IP solutions after missing LOI (Fig. 1b and 1c). This $\mathrm{C} 3$ difference results from IP solutions receiving more of a $\mathrm{C} 3$ increase from the b)
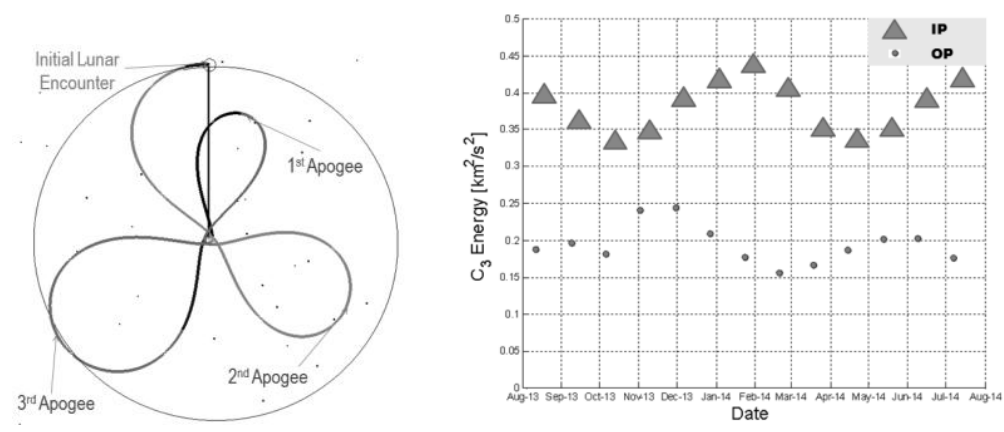

d)
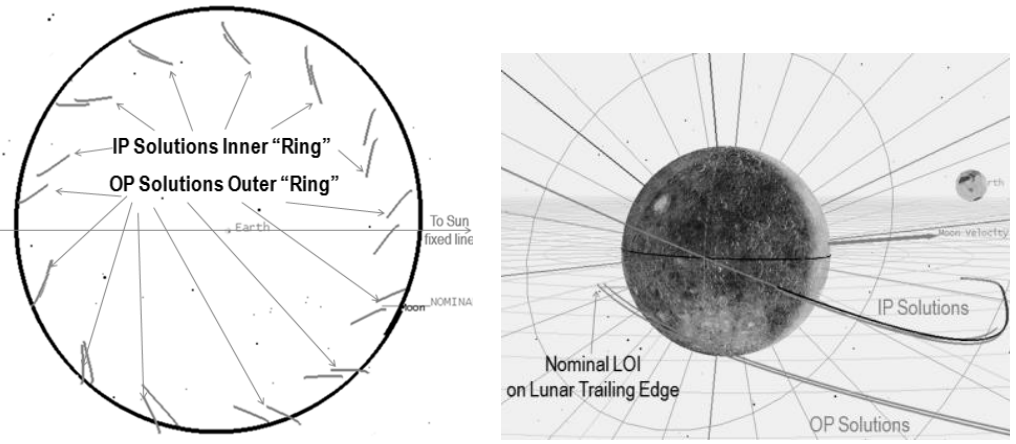

Figure 1. LADEE Nominal Trajectory and Effects of a Missed LOI Thereon. LADEE's Nominal Trajectory, view in Earth-centered, EarthMoon rotating frame (a); Earth Orbit Energy (C3) vs. LOI Date (b); post LOI miss states for LADEE from August 2013 to July 2014, view from north of $(c)$ and edge-on (d) the lunar orbit plane. 
Moon during the unintended trailing-edge flyby since such solutions lie in orbital planes less inclined to the lunar equatorial plane than that of OP solutions (Fig. 1d).

\section{Sun-Earth WSB Effects on Trajectory}

For all possible LADEE LOI miss states between August 2013 and July 2014, a maneuver was performed three days after the spacecraft recovered (e.g., "woke up" from "safe" mode) and was pointed in the orbit's anti-velocity direction to prevent escaping Earth to set up a return to lunar distance upon reaching subsequent perigee. The recovery maneuver $\Delta \mathrm{V}$ cost depended on the spacecraft's apogee location with on or near the Sun-Earth WSB. For posigrade orbits with quadrant II or IV apogee locations, solar perturbations ("crosswinds"13) increase the spacecraft's C3 while C3 is lowered by solar gravity for quadrant I or III apogee locations (Fig. 2a, from Ref. 14), ${ }^{13-15}$. The LADEE spacecraft's post LOI miss apogee would be posigrade and of higher energy than lunar orbit and thus the desired apogee location would be in quadrant I or III, since solar gravity would decrease $\mathrm{C} 3$ and the required recovery $\Delta \mathrm{V}$ cost. This cost difference is seen in Fig. $2 b$ and $2 d$, where the lowest energy transfers best utilized the Sun-Earth WSB to enable low energy lunar returns.

The WSB (or "crests of waves") transfers that yielded the lowest energy lunar return required $37 \mathrm{~m} / \mathrm{s}$ of $\Delta \mathrm{V}$ and seven months of flight time. The highest $\Delta \mathrm{V}$ transfers required $237 \mathrm{~m} / \mathrm{s}$ of recovery $\Delta \mathrm{V}$ and three months of flight time (similar three month transfers are analyzed by Itoh $^{16}$, and with solar gravity by Ishii ${ }^{17}$ and Tanabe, Itoh, et al. $^{18}$ ) with apogee locations in quadrant II and IV, as expected. Although the LADEE spacecraft could have performed the recovery maneuver for all analyzed cases, the science duration would be reduced for the highest recovery $\Delta \mathrm{V}$ solutions $(200 \mathrm{~m} / \mathrm{s}$ or more), which are the IP solutions (Fig. 2c). The $\Delta \mathrm{V}$ magnitude difference between these solutions results from the $\mathrm{C} 3$ difference previously seen in (Fig. $1 \mathrm{~b}$ and 1d): IP solutions attain a higher $\mathrm{C} 3$ than $\mathrm{OP}$ solutions via the (unintended) lunar flyby and thus require more $\Delta \mathrm{V}$ for lunar return.

a)

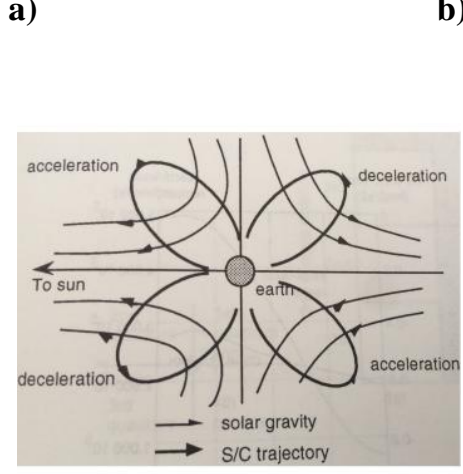

b)

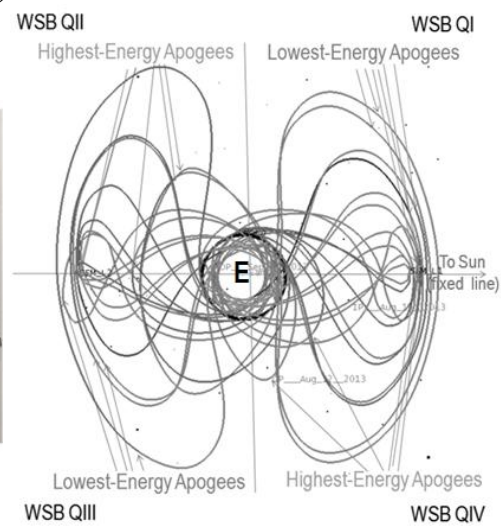

c)

d)

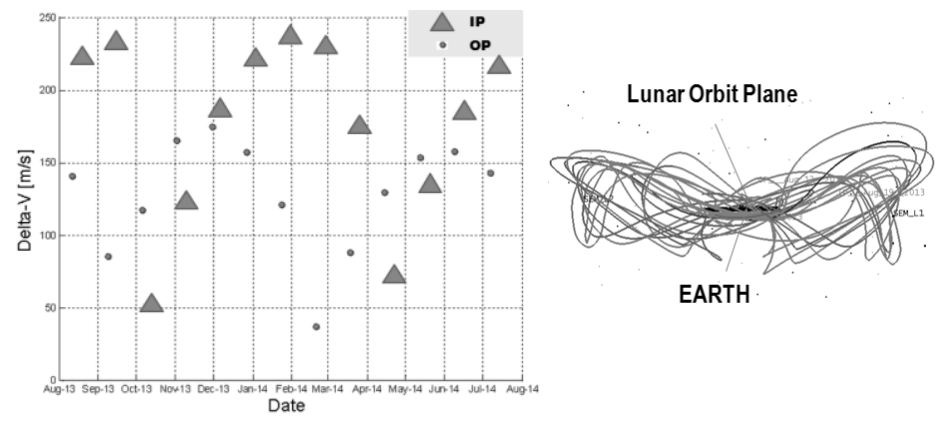

Figure 2. Effects of Sun-Earth WSB geometry on LADEE's Post LOI Miss Cases from Aug. 2013 to July 2014. General effects of solar gravity on spacecraft's orbit in Sun-Earth rotating frame, note Sun to the left (a); varying recovery $\Delta V$ costs to lunar distance shown for all LOI cases (c); LADEE's possible post LOI miss states shown after recovery $\Delta V$, in Sun-Earth rotating frame north of (b) and edge-on lunar orbit plane (d); note Sun to right.

a)

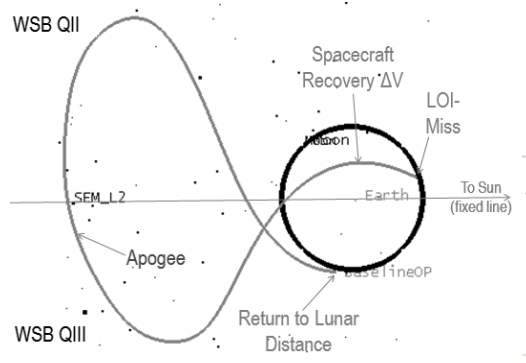

Figure 3. Single Loop Recovery Solutions. Shown in Earthcentered, Sun-Earth Rotating Frames. Lunar phasing is unfavorable for first solution (a); the second solution re-encounters the Moon upon its first perigee, but at the expense of a large $\Delta \mathrm{V}(359 \mathrm{~m} / \mathrm{s})$ performed at the preceding apogee $(b)$.

\section{First and Second Contingency Solutions}

Unfortunately, the single loop low energy returns discussed are generally not lunar periodic. For LADEE's baseline LOI case, the Moon would be on the opposite side of the Earth upon the spacecraft returning to lunar 
distance (first solution; Fig. 3a). Thus the $\Delta \mathrm{V}$ values in Fig. 2c represent minimum recovery $\Delta \mathrm{V}$ requirements for a given launch possibility, since more $\Delta \mathrm{V}$ is generally required for lunar phasing. This phasing $\Delta \mathrm{V}$ is performed at apogee to "counter" solar gravity in quadrant III, but at a $\Delta \mathrm{V}$ cost of 359 $\mathrm{m} / \mathrm{s}$ (second solution; Fig. 3b). Single loop solutions further constrained the baseline case via the $\Delta \mathrm{V}$ cost of changing the apogee altitude (and thus period) and the direction in which this altitude could be changed (i.e., a small increase in C3 would yield escape). Therefore multiple loop solutions were explored.

\section{Third and Fourth Contingency Solutions}

By implementing multiple Earth phasing orbits, more time is available for the spacecraft to change the spacecraft's arrival time at lunar distance. Multiple loops also allow an apogee that is fixed in inertial space to rotate in (Sun-Earth) rotating space. The rate of this natural apogee rotation depends on Earth's heliocentric period, thus it takes about one year to rotate apogee 360 degrees. Due to Earth's heliocentric motion, apogee rotates clockwise (CW) as viewed from north of Earth's orbit plane. This rotation rate was (approximately) observed (Fig. 4a), as LADEE's baseline LOI case would have established an apogee (altitude of 1.2 million $\mathrm{km}$ ) in WSB quadrant III with subsequent apogee locations rotating one full $\mathrm{CW}$ revolution in about 13 months.

LADEE's final apogee location was desired to be in quadrant II or IV, since either quadrant would yield a favorable lunar return via solar perturbations. (The first Sun-Earth WSB transfer was flown by the third International Sun-Earth Explorer spacecraft, which also flew multiple lunar flybys to reach its interplanetary destination, a comet $^{19,20}$, while the Hiten spacecraft later flew a WSB transfer that achieved the first ballistic lunar capture ${ }^{2}$.) Apogee would first rotate to quadrant II and a first attempt to solve this problem yielded the third solution (Fig. $4 \mathrm{~b}$ ); however, $80 \mathrm{~m} / \mathrm{s}$ of $\Delta \mathrm{V}$ was required at apogee for lunar re-encounter.

If apogee were to instead rotate to quadrant IV, only $25 \mathrm{~m} / \mathrm{s}$ of $\Delta \mathrm{V}$ would be needed for a lunar return, but the rotation would take ten months, yielding an undesirable total recovery duration of about one year. This quadrant IV (fourth) solution is shown in two frames: Earth inertial (Fig. 4c) and Sun-Earth rotating (Fig. 4a).

\section{Fifth Contingency Solution}

A more favorable lunar return is yielded by slightly decreasing the apogee altitude via a recovery $\Delta \mathrm{V}$ increase from $118 \mathrm{~m} / \mathrm{s}$ (third solution) to 140 $\mathrm{m} / \mathrm{s}$. The lunar re-encounter duration would be 167 days, compared to 233 days for the third solution. a)

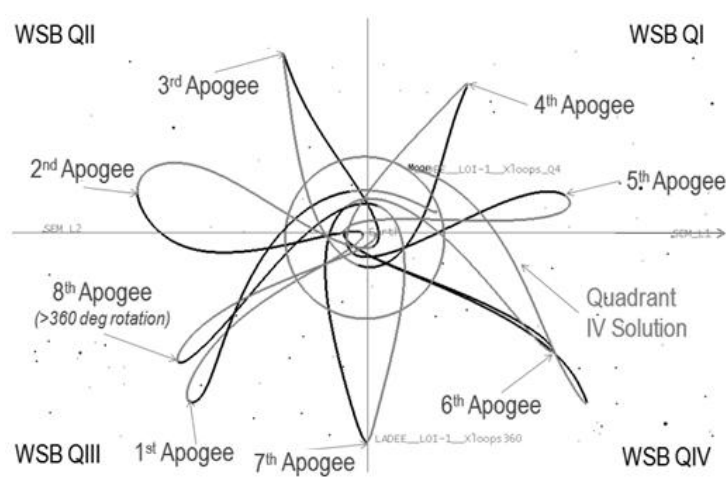

b)

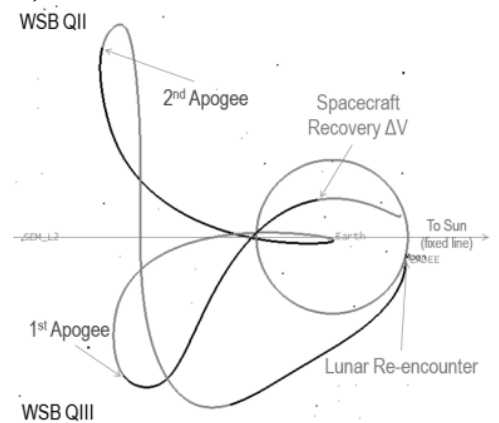

c)

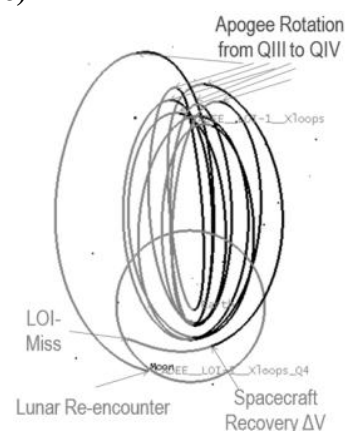

Figure 4. Apogee Rotation in Sun-Earth Rotating Frame. 360 degree apogee rotation in about 13 months (a); first attempt Quadrant II (third) solution in Sun-Earth rotating frame $(b)$; fourth solution, Quadrant IV solution in Earth-inertial frame (c).

a)

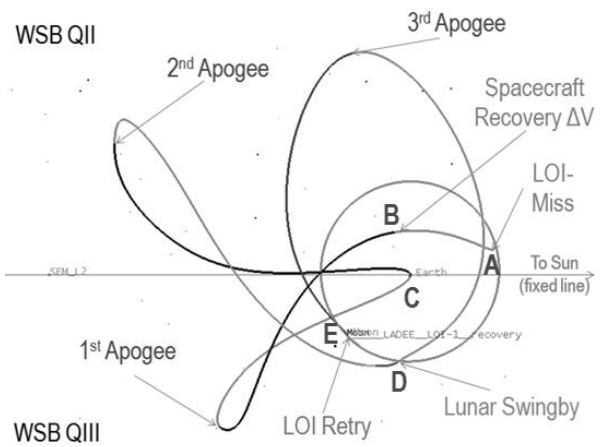

b)

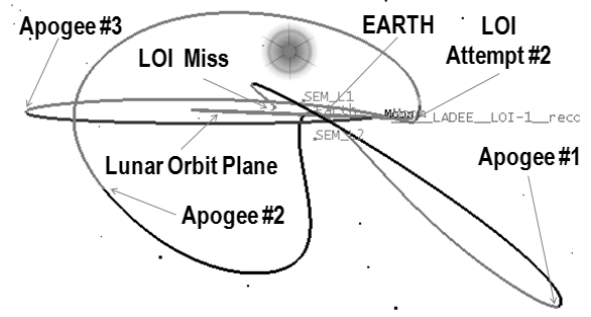

Figure 5. Selected Contingency Trajectory Design Solution. Fifth solution shown in Sun-Earth Rotating frames north of (a) and edge-on the lunar orbit plane (b).

4

American Institute of Aeronautics and Astronautics 
If the spacecraft missed LOI (Fig. 5a: A), the recovery maneuver would be performed three days later as a baseline (Fig. 5a: B); the first apogee would be in quadrant III followed by perigee at 2,600 km altitude (Fig. 5a: C). The spacecraft would perform a lunar re-encounter maneuver $(30 \mathrm{~m} / \mathrm{s}$ of $\Delta \mathrm{V})$ at its second apogee, at an altitude of 1.4 million $\mathrm{km}$ and located in quadrant II. This WSB transfer would arrive at the Moon with an arrival declination of 85 degrees (Fig. 5a: D, and Fig. 6a \& 6b). The corresponding orbit inclination range would be constrained between 79.6 and 98 degrees (Fig. $6 c)$, which is an unacceptable range for the science orbit. Therefore this lunar re-encounter would not be used for the LOI retry, but rather for a lunar flyby to change the orbital plane without the use of propellant. The flyby would be performed at a perilune altitude of $3,500 \mathrm{~km}$ to enter the required 157 degree inclination science orbit (Fig. 6b). Two months of duration was the primary cost of the flyby. The total recovery duration was seven months (Fig. 5a: E).

Despite a $140 \mathrm{~m} / \mathrm{s} \Delta \mathrm{V}$ requirement for the recovery maneuver, the LOI retry $\Delta \mathrm{V}(643 \mathrm{~m} / \mathrm{s})$ would be more than $150 \mathrm{~m} / \mathrm{s}$ less than the nominal LOI $\Delta \mathrm{V}$ since a Sun-Earth WSB lunar transfer trajectory would allow the spacecraft to approach the Moon at a lower relative speed as compared to the nominal transfer ${ }^{2,21}$. The total $\Delta \mathrm{V}$ required for this recovery was $848 \mathrm{~m} / \mathrm{s}$, or 13 $\mathrm{m} / \mathrm{s}$ less than the $\Delta \mathrm{V}$ required for nominal LOI. The three days assumed for this solution's recovery time was thus a conservative maximum. This (fifth) solution was ready to be flown by the LADEE spacecraft if needed.

\section{Effects of Varying Spacecraft Recovery Time}

The minimum recovery $\Delta \mathrm{V}$ requirement increases with the spacecraft recovery duration (Fig. 7c). A select few of these solutions fortuitously encounter the Moon upon reaching lunar distance and thus no $\Delta \mathrm{V}$ is needed at apogee for lunar phasing (i.e., "free-return"). For single loop solutions, a recovery duration of ten days yields such a lunar "free return" (Fig. 7a).

Free-return lunar encounters occur more frequently for double loop solutions since the range of lunar arrival dates is larger than that of single loops solutions. The two free-return double loop transfers occur when the recovery time is about three or ten days (Fig. 7b). Despite the baseline recovery time near a free-return value for the fifth solution, the lunar phasing $\Delta \mathrm{V}$ cost was non-zero $(50 \mathrm{~m} / \mathrm{s})$ since an additional lunar encounter was needed for the LOI retry. a)

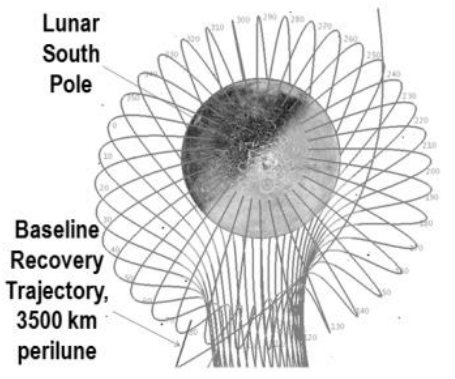

b)

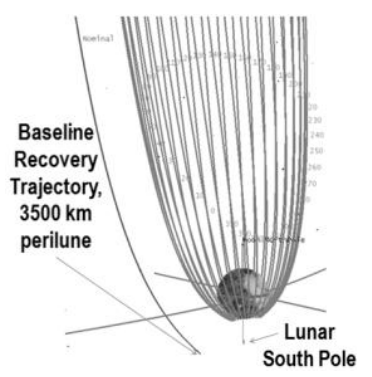

c)

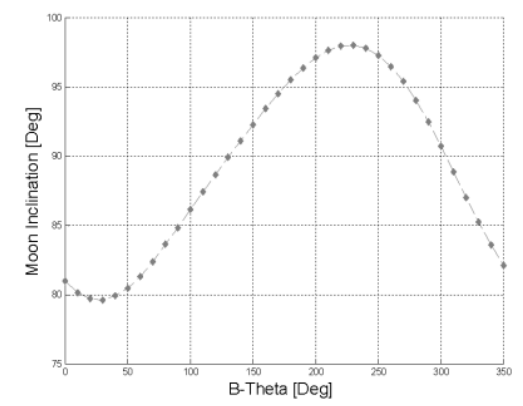

Figure 6. Effects of Arrival Declination on Lunar Orbit Inclination. Incoming lunar declination of 85 degrees, view normal to (a) and edge-on (b) lunar orbit plane; lunar orbit inclination shown for 360 degree B-theta range (c).

a)

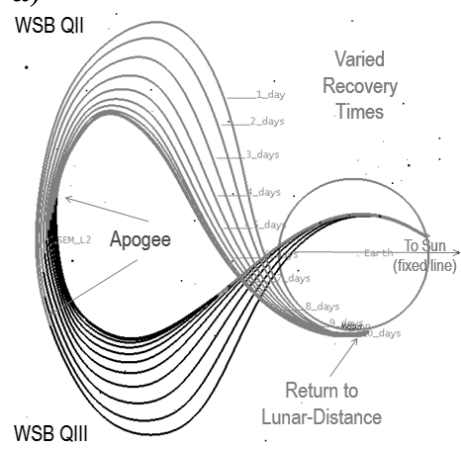

c)

b)

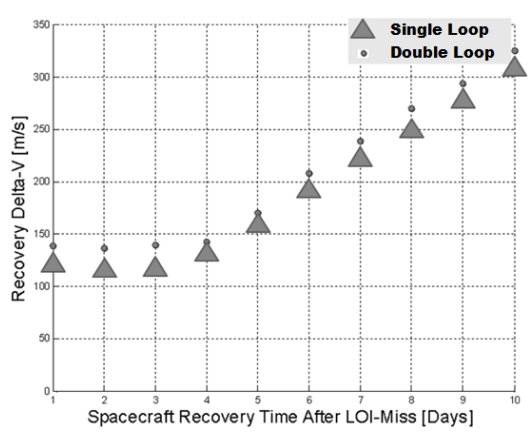

d)

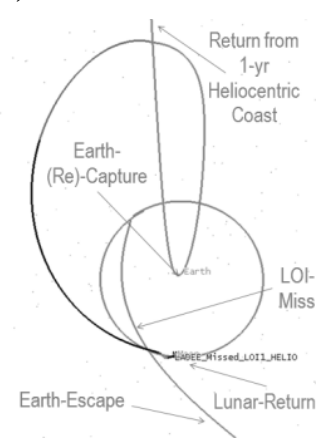

Figure 7. Lunar Return WSB Transfers and Recovery Duration vs. Recovery $\boldsymbol{\Delta} \mathbf{V}$. Single $(a)$ and double loop $(b)$ WSB transfer solutions and associated recovery $\Delta V$ costs for varying recovery durations (c); heliocentric return to Moon via reverse WSB transfer, shown in Earth inertial frame (d).

5

American Institute of Aeronautics and Astronautics 
From three to ten days of recovery time the mission could have been salvaged if the science duration was reduced from three months to one; less science duration would allow for the transfer of more than $65 \mathrm{~m} / \mathrm{s}$ of station-keeping $\Delta \mathrm{V}$ to the recovery $\Delta \mathrm{V}$ budget. After ten days, the mission could have been salvaged if the operational orbit changed to elliptical after a one year heliocentric lunar return via a reverse WSB transfer (Fig. 7d). Again, $\Delta \mathrm{V}$ would be reallocated to the recovery $\Delta \mathrm{V}$ budget from LOI $\Delta \mathrm{V}$ savings (elliptical vs. circular). Although an elliptical orbit would not achieve LADEE's science goals, it could have instead enabled the demonstration of the deep space laser communication system.

\section{LOI Underburn and Overburn Possibilities}

If the LADEE spacecraft's LOI maneuver ended prematurely, there were four types of recovery possibilities: 1) Lunar capture (minimum LOI $\Delta \mathrm{V}=$ $283 \mathrm{~m} / \mathrm{s}$ ) with no recovery maneuver required; higher LOI $\Delta \mathrm{V}$ values also yield lunar capture (up to the nominal value of about $800 \mathrm{~m} / \mathrm{s}$ to achieve a $587 \mathrm{~km}$ circular orbit); 2) Lunar capture (LOI $\Delta \mathrm{V}=250 \mathrm{~m} / \mathrm{s}$ ) with a recovery maneuver $(\Delta \mathrm{V}=37 \mathrm{~m} / \mathrm{s})$ required at first apolune (Fig. 8a); 3) Lunar re-encounter and LOI reattempt 25 days after the partial LOI failure (LOI $\Delta \mathrm{V}=200 \mathrm{~m} / \mathrm{s})$; the recovery maneuver $(\Delta \mathrm{V}=78 \mathrm{~m} / \mathrm{s})$ would be performed 11 days after the LOI underburn (Fig. 8b); 4) LOI reattempt (LOI $\Delta \mathrm{V}=157 \mathrm{~m} / \mathrm{s}$ ) would occur 168 days after the partial LOI failure; the recovery maneuver $(66 \mathrm{~m} / \mathrm{s}$ of $\Delta \mathrm{V})$, would occur at the second apogee 118 days after the LOI underburn, seen in Fig. 8c. LOI $\Delta \mathrm{V}$ underburns less than $157 \mathrm{~m} / \mathrm{s}$ would perform a recovery maneuver three days after the first LOI and fly the fifth solution type (Fig. 5).

An LOI overburn of up to $905 \mathrm{~m} / \mathrm{s}$, or $12.5 \%$ more than the $\Delta \mathrm{V}$ to enter a $587 \mathrm{~km}$ circular orbit, could have been tolerated (Fig. 8d). The $\Delta \mathrm{V}$ cost of raising perilune to $250 \mathrm{~km}$ (not $587 \mathrm{~km}$ ) would be $50 \mathrm{~m} / \mathrm{s}$.

\section{Multiple LOI Maneuver Misses}

If the LADEE spacecraft (fully) missed two LOI maneuvers, a recovery maneuver of $81 \mathrm{~m} / \mathrm{s}$ would be performed three days after missing the second LOI. This would result in a 14 day return for the third LOI attempt (Fig. 9a) and require a total recovery $\Delta \mathrm{V}$ of $924 \mathrm{~m} / \mathrm{s}$ (feasible for the LADEE spacecraft with a reduced science duration).

If the third LOI maneuver was missed, a fourth LOI attempt could occur 50 days later (Fig. 9b). The spacecraft would perform the recovery maneuver (96 $\mathrm{m} / \mathrm{s}$ of $\Delta \mathrm{V}$ ) at apogee (about one month after the third LOI miss). Such a predicament would yield a total recovery $\Delta \mathrm{V}$ requirement of $1,020 \mathrm{~m} / \mathrm{s}$, which could have been performed by the LADEE spacecraft if the science duration was reduced to about one month. a)

b)

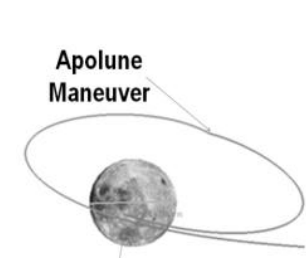

LOIUnderburn (Direct Capture)

c)

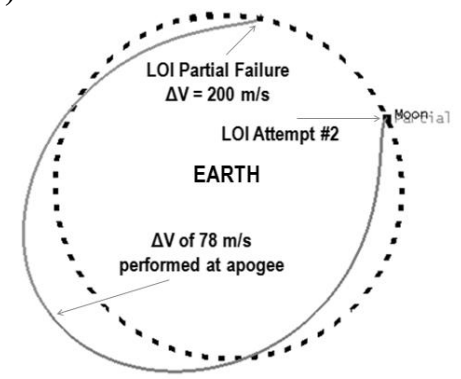

d)

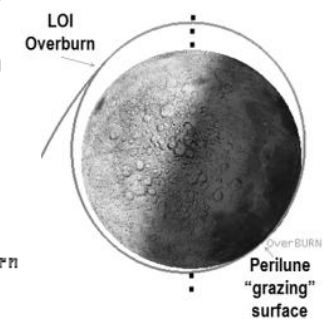

Figure 8. LOI Maneuver Failure Types. Lunar capture with $37 \mathrm{~m} / \mathrm{s}$ recovery $\Delta V$ performed at apolune, 1.7 days after first LOI attempt (a); failed lunar capture after 200 $\mathrm{m} / \mathrm{s} \mathrm{LOI} \triangle \mathrm{V}(\mathrm{b}) ; 157 \mathrm{~m} / \mathrm{s} \mathrm{LOI}$ (underburn) would yield a 168 day WSB transfer back to Moon (c); overburn of $12.5 \%$ is the maximum tolerable before lunar impact $(d)$.

a)

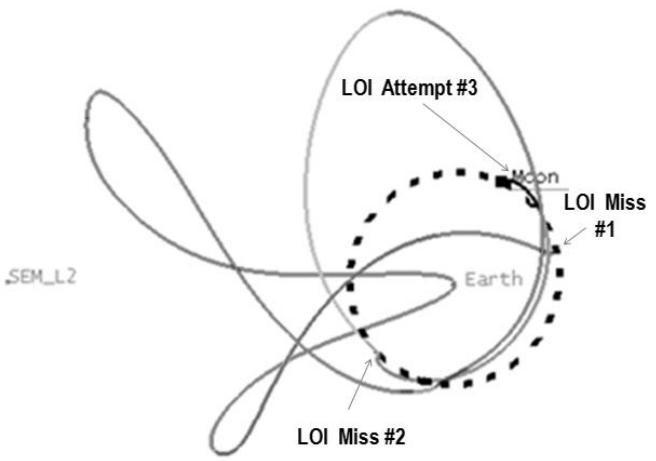

b)

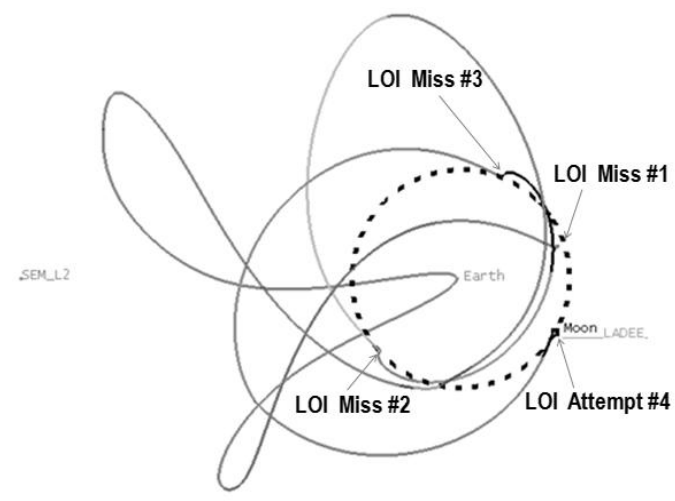

Figure 9. Multiple LOI Maneuver (Full) Misses.

Third LOI attempt shown ten days after second LOI miss (a); fourth LOI attempt shown 50 days after a third LOI miss (b). Both shown in Sun-Earth rotating frames. 


\section{Conclusion}

The fifth solution presented would be able to recover the LADEE spacecraft as flown for the baseline recovery case (Fig. 5). A six month period in the recovery $\Delta \mathrm{V}$ requirement (vs. LOI date) was observed throughout a one year launch period due to varying apogee locations on or near the Sun-Earth WSB (Fig. 1b, 2c). It was seen that the LADEE spacecraft could have recovered for all analyzed launch opportunities throughout the one year of launch possibilities. As flown, LADEE could have recovered into its required science orbit if a recovery $\Delta \mathrm{V}$ was performed within ten days of missing LOI (albeit with a reduced science duration). Generally, the total $\Delta \mathrm{V}$ requirement increased with recovery time due to the increased cost of the recovery maneuver and sometimes significant $\Delta \mathrm{V} \operatorname{cost}$ to phase with the Moon. Other recovery types shown include: underburns, overburns, and multiple (three) full LOI misses. Finally, the apogee rotation design element seen in Fig. 4a can be extended to other systems (e.g., SunVenus, Sun-Mars, et al.) to enable a low energy planetary return and/or escape for a spacecraft.

\section{Acknowledgments}

The author thanks NASA ARC management and LADEE team members Mike Loucks (Trajectory Design Lead), John Carrico (Orbit Determination Lead), Ken Galal (Guidance, Navigation, and Control Lead), and Butler Hine (Project Manager) for their appreciated support. Additionally, the author thanks the NASA ARC Technical Library staff, notably Daniel Pappas and Kathleen Ponce for consistently providing timely and helpful support.

\section{References}

${ }^{1}$ Belbruno, E., "Lunar Capture Orbits, a Method of Constructing Earth-Moon Trajectories and the Lunar GAS Mission," AIAA/DGLR/JSASS International Elec. Propl. Conf., AIAA-87-1054, Reston, VA May 1987. (No page numbers.)

${ }^{2}$ Belbruno, E., and Miller, J., “A Ballistic Lunar Capture Trajectory for the Japanese Spacecraft Hiten,” Jet Propulsion Laboratory Interoffice Memorandum 312/90.4-1731-EAB, June 15, 1990.

${ }^{3}$ Belbruno, E., "Examples of the Nonlinear Dynamics of Ballistic Capture and Escape in the Earth-Moon System," AIAA-90-2896, AIAA/AAS Astrodynamics Specialist Conference, Portland, OR, Aug. 20-22, 1990. (Conference Proceedings not found.)

${ }^{4}$ Belbruno, E., "Through the Fuzzy Boundary: A New Route to the Moon," Planetary Report, Planetary Society, Pasadena, CA, Vol. 7, No. 3, pp. 8-10, May-June, 1992.

${ }^{5}$ Hill, G., "Researches in the Lunar Theory," American Journal of Mathematics, Johns Hopkins University, Baltimore, MD, Vol. 1, No 1, pp. 5-26, 1878.

${ }^{6}$ Darwin, G., "Periodic Orbits," Acta Mathematica, Springer, New York, NY, pp. 99-242, 1897.

${ }^{7}$ Moulton, 1914, "Celestial Mechanics," ㅇ 1914 by The MacMillan Co., Thirteenth Edition, New York, NY, p. $281,1959$.

${ }^{8}$ Wintner, A., "The Analytical Foundations of Celestial Mechanics," (C) 1941 by Princeton University Press, Second Printing, Princeton, NJ, pp. 120, 1947.

${ }^{9}$ Llibre, J. and Martinez, R., "Tranversality of the Invariant Manifolds Associated to the Lyapunov Family of Periodic Orbits near $\mathrm{L}_{2}$ in the Restricted Three-Body Problem," Journal of Differential Equations, Springer, New York, NY, Vol. 58, pp. 104$156,1985$.

${ }^{10}$ Lundberg, J., Szebehely, V., Nerem, R.,, and Beal, B., "Surfaces of zero velocity in the restricted problem of three bodies." Celestial Mechanics, Springer, New York, NY, Vol. 36, No. 2, pp. 191-205, 1985.

${ }^{11}$ Belbruno, E., Gidea, M., and Topputo, F., "Weak Stability Boundary and Invariant Manifolds," J. Applied Dynamical Systems, SIAM, Philadelphia, PA, Vol. 9, No. 3, pp. 1061-1089, 2010.

${ }^{12}$ Loucks, M. (2013, Sep. 12). Phasing Loops and the LADEE Trajectory. AstrogatorsGuild.com. Retrieved January 2, 2014, from http://astrogatorsguild.com/?p=814 [cited 13 June 2014].

${ }^{13}$ Penzo, P., Bender, D., and Cassell, C., "Multiple Lunar Swingbys for Small Body and Planetary Missions," Advances in the Astronautical Sciences, Univelt, San Diego, CA, Vol. 89, Part I, pp. 317-330, 1995.

${ }^{14}$ Yamakawa, H., Kawaguchi, J., Ishii, N., and Matsuo, H., "On Earth-Moon Transfer Trajectory with Gravitational Capture," Advances in the Astronautical Sciences, Univelt, San Diego, CA, Vol. 85, Part I, pp. 397-416, 1993.

${ }^{15}$ Biesbroek, R., and Janin, G., "Ways to the Moon?," European Space Agency Bulletin 103 URL: http://www.esa.int/esapub/bulletin/bullet103/biesbroek103.pdf [cited 21 June 2014], Paris, France, pp. 92-99, August, 2000.

${ }^{16}$ Itoh, Y., "Orbital Transfers to $\mathrm{L}_{4}$ and $\mathrm{L}_{5}$ in the Earth-Moon System," Bachelor's Thesis, University of Tokyo, March 1981.

${ }^{17}$ Ishii, N., "Transfers to Libration Points Using a Lunar Swingby," Bachelor's Thesis, University of Tokyo, March 1982.

${ }^{18}$ Tanabe, T., Itoh, Y., Ishii, N, and Yokota, H., "Visiting Libration Points in the Earth-Moon System Using a Lunar Swingby," International Symposium on Space Technology and Science, AGNE Publishing, Inc., pp. 433-438, 1982.

${ }^{19}$ Farquhar, R., Muhonen, D., and Richardson, D., "Mission Design for a Halo Orbiter of the Earth," Journal of Spacecraft and Rockets, AIAA, Reston, VA, Vol. 14, No. 3, pp. 170-177, March 1977.

${ }^{20}$ Farquhar, R, Muhonen, D., and Church, L., "Trajectories and Orbital Maneuvers for the ISEE-3/ICE Comet Mission," Journal of the Astronautical Sciences, Univelt, San Diego, CA, Vol. 33, No. 3, pp. 235-254, July-September 1985.

${ }^{21}$ Yamakawa, H., Kawaguchi, J., Ishii, N., and Matsuo, H., "A Numerical Study of Gravitational Capture Orbit in the Earth-Moon System," Advances in the Astronautical Sciences, Univelt, San Diego, CA, Vol. 79, Part II, pp. 1113-1132, 1992. 